\title{
Review \\ Year in review in Critical Care, 2004: sepsis and multi-organ failure
}

\author{
James M O'Brien, Jr ${ }^{1}$, Naeem A Ali ${ }^{1}$ and Edward Abraham²
}

\begin{abstract}
${ }^{1}$ Assistant Professor, Division of Pulmonary, Critical Care and Sleep Medicine, The Ohio State University Medical Center, Columbus, Ohio, USA ${ }^{2}$ Roger Sherman Mitchell Professor of Pulmonary and Critical Care Medicine, Vice Chair, Department of Medicine, Head, Division of Pulmonary Sciences and Critical Care Medicine, University of Colorado at Denver and Health Sciences Center, Denver, Colorado, USA
\end{abstract}

Corresponding author: James M O'Brien, Jr, obrien-2@medctr.osu.edu

Published online: 23 May 2005

This article is online at http://ccforum.com/content/9/4/409

(c) 2005 BioMed Central Ltd

\section{Introduction}

During 2004, Critical Care published a broad range of original research focused on sepsis and the multi-organ dysfunction syndrome (MODS). These studies included epidemiologic surveys, assessments of the pathogenesis of the syndrome, analyses of prognostic variables in affected patients, and new therapeutic modalities.

\section{National rates of sepsis}

In an attempt to determine country-specific rates of sepsis, three studies estimated the incidence of sepsis in the Netherlands [1], in Norway [2], and in Brazil [3].

van Gestel and colleagues [1] performed a cross-sectional survey of patients in 47 Dutch intensive care units (ICUs) using American College of Chest Physicians/Society of Critical Care Medicine consensus criteria for sepsis, severe sepsis, or septic shock [4]. The authors estimated an incidence of severe sepsis of 0.54 cases/1000 population per year, indicating that severe sepsis accounts for $0.61 \%$ of all hospital admissions and for $11 \%$ of all ICU admissions in the Netherlands.

Flaatten [2] used International Classification of Diseases (ICD-10) codes in a national dataset in Norway to detect episodes of sepsis. They found an incidence of sepsis of 1.49 cases/1000 population. Among hospitalized patients, the sepsis and severe sepsis rates were 9.5 and 3.0 cases per 1000 admissions, respectively. Incidence and mortality rates rose in an age-dependent fashion.

Silva and colleagues [3] described the findings of the Brazilian Sepsis Epidemiological Study, a prospective cohort study of consecutive adult admissions to five Brazilian ICUs. The rates of sepsis, of severe sepsis, and of septic shock were 305,174 , and 147 cases per 1000 ICU admissions,
Critical Care 2005, 9:409-413 (DOI 10.1186/cc3728)

respectively. Approximately two-thirds of septic patients met diagnostic criteria on admission, with the remainder diagnosed on subsequent days. The mortality rates of patients with the systemic inflammatory response syndrome, sepsis, severe sepsis, and septic shock were $24.2 \%, 33.9 \%$, $46.9 \%$, and $52.2 \%$, respectively. Survival was lower in the septic patients compared with those patients without sepsis.

\section{Diagnosis and transmission of infections}

Controversy exists in the approach to diagnosing ventilatorassociated pneumonia. Camargo and colleagues [5] assessed the utility of tracheal aspirates to diagnose ventilator-associated pneumonia in mechanically ventilated patients. Qualitative culture had the highest sensitivity in the diagnosis of ventilator-associated pneumonia but had poor specificity when compared with quantitative analysis. The bacterial yield was affected by antibiotic use. Overall, the authors concluded that quantitative assessment of tracheal secretions is superior to qualitative measures for diagnosing ventilator-associated pneumonia.

Agvald-Ohman and colleagues [6] investigated the colonization and transmission rate of coagulase-negative staphylococci among 20 intubated patients. On at least one occasion, $85 \%$ of subjects were colonized with coagulasenegative staphylococci and 70\% appeared to have been involved in at least one transmission event of coagulasenegative staphylococci. The authors suggested that a surveillance program measuring colonization rates might provide greater insight than simply documenting clinical infections with coagulase-negative staphylococci.

In a detailed case report, Naija and colleagues [7] described a patient with postoperative meningitis due to Pseudomonas with serial assessments of cerebrospinal fluid from ventricular and lumbar drains. Lumbar inflammation was consistently 
greater (higher leukocyte count, higher protein, and lower glucose) than that seen from ventricular drains. The authors suggested that diagnosis based on ventricular cerebrospinal fluid may lead to delays in recognition.

\section{Markers and mediators of sepsis and MODS}

Attention has been focused on biomarkers that may facilitate diagnosing sepsis. In a large study of emergency department patients, Chan and colleagues [8] tested whether procalcitonin and C-reactive protein could discriminate between patients with and without bacterial infections. Among patients requiring admission for suspected infection, procalcitonin was significantly higher in those patients with bacteremia and septic shock. However, procalcitonin did not discriminate patients with less severe infections from those without infection. C-reactive protein did not provide information about the severity of the infection but did discriminate between those with and without an infection.

Castelli and colleagues [9] tested whether the association between inflammatory markers and sepsis is specific or is simply a reflection of the severity of critical illness. The authors compared 150 critically ill patients with organ failure from either infectious (sepsis) or non-infectious (trauma) causes. The mean $\mathrm{C}$-reactive protein and procalcitonin levels were significantly higher in septic patients than in patients with trauma. C-reactive protein did not have an association with the severity of organ dysfunction in either group and did not increase further in trauma patients once an infection developed. Procalcitonin levels were associated with greater severity of illness in septic patients, but not in trauma patients. In the trauma group, acquired infections were accompanied by a rise in procalcitonin. The authors suggest that procalcitonin may allow early identification of trauma patients who develop infection.

In a secondary analysis of patients with severe sepsis enrolled in a phase 3 study of activated protein C, Kinasewitz and colleagues [10] reported the extent of coagulation abnormalities. Markers of coagulation and endothelial function were assessed at baseline in all patients and were assessed serially in patients receiving placebo. Coagulopathy was nearly universal at presentation during severe sepsis, while several inflammatory cytokines were unmeasurable in a significant proportion of patients. In the placebo-treated patients, serial measurements of hemostatic markers revealed that non-survivors had a greater level of coagulopathy at presentation and demonstrated less normalization over the first 7 days. This relationship persisted despite infection with either Gram-negative or Gram-positive organisms. This study emphasizes the profound coagulopathy that occurs in severe sepsis.

While anemia is common, little is known about erythropoietin levels in sepsis. Tamion and colleagues [11] measured serial renin and erythropoietin levels over 48 hours in 50 septic shock patients. The erythropoietin levels were significantly higher among non-survivors compared with survivors of septic shock. Renin levels did not vary. Unlike the normal relationship seen in survivors of septic shock, erythropoietin levels fluctuated independently of hemoglobin in nonsurvivors. After multivariate analysis, erythropoietin and $\mathrm{pH}$ were independently associated with mortality. This study suggests that sepsis-induced hematopoietic dysregulation can be a marker of poor outcome.

Studies in septic shock patients with myocardial depression have indicated that levels of c-terminal active brain natriuretic protein are elevated [12], but the $n$-terminal portion brain natriuretic protein has not been tested. Chua and Kang-Hoe [13] showed that $n$-terminal portion brain natriuretic protein levels were elevated at presentation in six patients with septic shock and myocardial dysfunction. This suggests that sepsisinduced myocardial dysfunction is an alternate reason for elevated brain natriuretic protein levels in sepsis.

Patients with alcoholism appear to develop sepsis more frequently and to have worse outcomes than non-alcoholics [14]. In a cohort of patients with septic shock from peritonitis or pneumonia, Von Dossow and colleagues [15] compared the inflammatory cytokine profiles between those patients with and without alcoholism. At the onset of infection, proinflammatory cytokines (e.g. IL-8, IL-6, IL-1 $\beta$ ) were suppressed in patients with alcoholism compared with nonalcoholics. Additionally, the rise in proinflammatory cytokines seen in non-alcoholics with the development of septic shock was absent in the alcoholic patients. This suggests that alcoholism may blunt the early proinflammatory response to infection in patients with septic shock.

\section{Insulin and glycemia in sepsis}

Rusavy and colleagues [16] compared energy expenditure and glucose uptake in response to insulin in non-diabetic patients with severe sepsis to healthy controls. In hemodynamically stable fasting patients (days 3-7 after sepsis onset), a two-step insulin clamp protocol was used to achieve two levels of hyperinsulinemia while maintaining normoglycemia. The basal energy expenditure was significantly higher in septic patients but the insulin-induced increase was not as dramatic as that seen in the controls. The same response was seen regarding glucose uptake, a composite measure of glucose storage and oxidation. Further study demonstrated that insulin increased glucose storage and oxidation in control subjects while it increased only oxidation in the septic patients. This suggests that the metabolic response to insulin is different in septic patients from that in normal controls.

Vriesendorp and colleagues [17] performed a retrospective cohort study of patients undergoing esophagectomy to determine whether glucose control was associated with the postoperative course. Glucose management was not 
standardized, but early enteral feeding (within 24 hours) was performed in all patients and insulin was encouraged in hyperglycemia (e.g. greater than $12 \mathrm{mmol} / \mathrm{l}$ ). After adjustment for multiple surgical and patient variables, the increased mean glucose was not associated with an increased length of stay or with the occurrence of infection. The authors suggested that their findings question the relevance of intensive glucose control in all critically ill patients and may have a more important role in those with established vascular disease.

\section{Sepsis in cancer}

In a retrospective cohort study, Williams and colleagues [18] used International Classification of Diseases (ICD-9) codes to identify severe sepsis among patients with cancer in six state hospital discharge databases. After adjusting for age and gender, the cancer population was almost four times more likely to be hospitalized with severe sepsis (relative risk, 3.96; 95\% confidence interval, 3.94-3.99) than the noncancer population. The authors estimated 126,200 cases of severe sepsis annually in cancer patients, with the highest risk in those with myeloid leukemia. The overall hospital mortality was $52 \%$ higher for severe sepsis patients with cancer than for severe sepsis patients without cancer. Nearly $10 \%$ of the annual cancer-related deaths and $14 \%$ of the cancer-associated hospitalization costs were due to severe sepsis. An age-dependent increase in the incidence and mortality of severe sepsis was observed in the non-cancer population, but not in cancer patients.

Soares and colleagues [19] compared the performance of five general severity of illness scores in predicting hospital mortality with a cancer-specific score in a cohort of cancer patients requiring ICU admission. The authors evaluated patients in a dedicated oncologic ICU where almost one-half of patients are admitted emergently and where 20\% have sepsis. The simplified acute physiology score (SAPS2) predicted mortality most accurately. However, the calibration of all scores was poor. The general models underestimated hospital mortality, while the cancer-specific model overestimated it. Changing demographic of patients with malignancy and newer therapies may make existing mortality prediction models obsolete.

\section{Animal studies in the management of sepsis and MODS}

Lagoa and colleagues [20] studied dogs to determine whether early resuscitation improved mucosal blood flow and mesenteric oxygen metabolism. Using an intravenous Escherichia coli model, the animals were randomly assigned to receive no fluid resuscitation or to receive large-volume crystalloid resuscitation. The bacterial infusion produced predictable hemodynamic and metabolic changes, marked decreases in mesenteric blood flow and increased measures of mesenteric hypoxia. While the majority of systemic variables were improved by resuscitation, the mesenteric blood flow was only partly increased by resuscitation and other markers of mesenteric perfusion were unaffected by volume infusion. Fluid replacement prevented a continued rise in the difference between gastric mucosal and arterial $\mathrm{PCO}_{2}\left(\mathrm{CO}_{2}\right.$ gap) values but did not restore levels to those seen at baseline. This suggests a disparity between the responses in hemodynamic measures and mucosal perfusion after volume resuscitation.

Modulation of the immune response to bacterial products has been the subject of numerous investigations in sepsis. Goscinski and colleagues [21] investigated the ability of tobramycin and ceftazidime to alter the inflammatory response to endotoxin infusion. Prior to endotoxin administration, piglets received intravenous tobramycin, ceftazidime, or placebo. The expected physiologic changes occurred with endotoxin infusion. There were no significant differences in circulatory, respiratory, or hematologic variables, or in endotoxin levels between the groups. After 3 hours, IL-6 levels decreased to a greater degree in the antibiotic groups than in the placebo animals but tumor necrosis factor alpha levels were not affected. This suggests that while tobramycin and ceftazidime do not neutralize endotoxin, they may have an effect on IL-6.

Vascular permeability, as measured by extravascular lung water, increases before changes in oxygenation in animal models of acute lung injury. Current methods require a double injection indicator to measure extravascular lung water. A single thermodilution technique is technically easier to perform. In a sheep model of acute lung injury, Kirov and colleagues [22] used a single thermodilution technique to assess extravascular lung water. The extravascular lung water measured by the single thermodilution technique was well correlated with gravimetric assessment at the postmortem examination $(r=0.85)$. However, it consistently overestimated the postmortem lung weight raising concerns about its specificity for diagnosing excess lung water.

\section{Human studies in the management of sepsis and MODS}

Because of its antioxidant and anti-inflammatory properties [23], $N$-acetylcysteine is an attractive agent for modulating the response to sepsis. Hein and colleagues [24] used a variety of techniques to assess liver perfusion, liver function, and lactate production after intravenous $\mathrm{N}$-acetylcysteine $(150 \mathrm{mg} / \mathrm{kg})$ administration in five patients with respiratory failure and septic shock. After treatment with $\mathrm{N}$-acetylcysteine, liver perfusion and hepatic function increased and liver lactate intensity decreased. This suggests that $\mathrm{N}$-acetylcysteine increased hepatic perfusion and improved hepatic oxidative metabolism. Emet and colleagues [25] performed a randomized controlled trial of early $N$-acetylcysteine infusion in patients with severe sepsis. Patients in the $N$-acetylcysteine group $(n=27)$ received an intravenous bolus followed by a continuous infusion for 
6 hours, and control subjects $(n=26)$ received placebo. The hospital mortality, the duration of mechanical ventilation, and the length of ICU stay were no different between the two groups. There were no significant differences between the groups in hemodynamic measures, in gastric $\mathrm{pH}$, or in inflammatory markers. No significant adverse events were noted. The authors concluded that the use of $\mathrm{N}$-acetylcysteine in patients with severe sepsis is not currently supported but further investigation might be warranted.

Vasoactive arachidonic acid metabolites, especially thromboxane $A_{2}$ and prostacyclin, may play a role in the pathogenesis of septic shock and MODS [26]. Memis and colleagues [27] conducted a randomized, placebo-controlled trial of lornoxicam, an inhibitor of cyclooxygenase, in patients with severe sepsis $(n=40)$. One-half of the patients received lornoxicam ( $8 \mathrm{mg}$ intravenously every 12 hours for six doses) and one-half received placebo. There were no differences between the two groups with regard to physiologic measures, to arterial blood gas values, or to levels of inflammatory markers. There was no benefit of lornoxicam on ICU mortality, on number of ventilator days, or on ICU length of stay. No adverse events were noted.

In supporting the respiratory system of patients with acute respiratory distress syndrome, positive end expiratory pressure is often employed. Bruhn and colleagues [28] investigated the effect of a range of positive end expiratory pressure levels on gastric mucosal perfusion. Eight adult patients with acute respiratory distress syndrome were included. Pressure-volume curves measured by the airway occlusion technique defined ideal positive end expiratory pressure. Subjects received positive end expiratory pressure levels of $10 \mathrm{cmH}_{2} \mathrm{O}, 15 \mathrm{cmH}_{2} \mathrm{O}, 20 \mathrm{cmH}_{2} \mathrm{O}$ and ideal positive end expiratory pressure for four consecutive 30-min periods. During the study, the majority of hemodynamic measures did not vary but the mean airway pressure and the $\mathrm{PaO}_{2} / \mathrm{FiO}_{2}$ ratio increased with increasing positive end expiratory pressure. Overall, no significant change in the $\mathrm{CO}_{2}$ gap or cardiac output was found at any of the study periods, but individual variations were noted.

Some studies suggest that early surgical intervention is associated with poorer outcomes in severe acute pancreatitis [29]. To explore this association, De Waele and colleagues [30] reported their experience with 124 patients with severe acute pancreatitis at a hospital in Belgium. Forty-five percent underwent surgery, and $39.2 \%$ of these had early surgery (within 12 days of diagnosis). Using logistic regression, the authors found that early surgery was not independently associated with mortality, once adjusted for age, for sequential organ failure assessment score at the time of surgery, and for the presence of sterile necrosis. The authors suggested that the reported association between early surgical intervention and mortality may be due to a lack of adequate risk-adjusting.

\section{Recovery from sepsis and MODS}

Kerbaul and colleagues [31] described a cohort of 15 patients with ICU-acquired weakness following open heart surgery complicated by sepsis. The eight survivors were followed for neurologic recovery for up to 1 year. Twenty-five percent could not ambulate independently at 1 year followup. This was predicted by the combination of muscle and nerve pathology on biopsy and the absent nerve conduction on electrophysiologic testing. This combination, if prospectively confirmed, may identify a group of patients with a high risk for long-term disability.

Granja and colleagues [32] compared health-related quality of life between patients admitted to a medical/surgical ICU in Portugal for severe sepsis or septic shock with those patients admitted for reasons other than severe sepsis. Among the septic respondents, 33\% reported problems with ambulation, $24 \%$ reported problems with self-care, $46 \%$ had problems with self-care, $36 \%$ had pain or discomfort, and $44 \%$ were anxious or depressed 6 months after the ICU stay. These measures were similar to the non-septic group. At the time of assessment, $33 \%$ of septic patients and $42 \%$ of the comparison patients reported their current health state was worse compared with 12 months prior to the assessment.

\section{Physician attitudes and awareness of sepsis}

Poeze and colleagues [33] surveyed physicians' attitudes about sepsis and their awareness of American College of Chest Physicians/Society of Critical Care Medicine consensus conference diagnostic criteria. The majority felt that sepsis was a leading cause of ICU mortality, that sepsis carried a significant financial burden, and that sepsis was a challenging condition to treat. Despite the recognition of sepsis as an important disease, only $22 \%$ of intensivists and $5 \%$ of non-intensivists gave the consensus conference diagnostic criteria when defining sepsis. Only $17 \%$ of physicians agreed on any one definition of sepsis. Fever was the only sign mentioned by a majority of respondents as a requirement to confirm the diagnosis.

\section{Competing interests}

The author(s) declare that they have no competing interests.

\section{References}

1. van Gestel A, Bakker J, Veraart CP, van Hout BA: Prevalence and incidence of severe sepsis in Dutch intensive care units. Crit Care 2004, 8:R153-R162.

2. Flaatten H: Epidemiology of sepsis in Norway in 1999. Crit Care 2004, 8:R180-R184.

3. Silva E, Pedro MA, Sogayar AC, Mohovic T, Silva CL, Janiszewski M, Cal RG, de Sousa EF, Abe TP, de Andrade J, et al.: Brazilian Sepsis Epidemiological Study (BASES study). Crit Care 2004, 8:R251-R260.

4. Bone RC, Sprung CL, Sibbald WJ: Definitions for sepsis and organ failure. Crit Care Med 1992, 20:724-726.

5. Camargo LF, De Marco FV, Barbas CS, Hoelz C, Bueno MA, Rodrigues M, Jr, Amado VM, Caserta R, Martino MD, Pasternak J, Knobel E: Ventilator associated pneumonia: comparison between quantitative and qualitative cultures of tracheal aspirates. Crit Care 2004, 8:R422-R430. 
6. Agvald-Ohman C, Lund B, Edlund C: Multiresistant coagulasenegative staphylococci disseminate frequently between intubated patients in a multidisciplinary intensive care unit. Crit Care 2004, 8:R42-R47.

7. Naija W, Mateo J, Raskine L, Timsit JF, Lukascewicz AC, George $B$, Payen D, Mebazaa A: Case report: greater meningeal inflammation in lumbar than in ventricular region in human bacterial meningitis. Crit Care 2004, 8:R491-R494.

8. Chan YL, Tseng CP, Tsay PK, Chang SS, Chiu TF, Chen JC: Procalcitonin as a marker of bacterial infection in the emergency department: an observational study. Crit Care 2004, 8:R12R20.

9. Castelli GP, Pognani C, Meisner M, Stuani A, Bellomi D, Sgarbi L: Procalcitonin and $\mathrm{C}$-reactive protein during systemic inflammatory response syndrome, sepsis and organ dysfunction. Crit Care 2004, 8:R234-R242.

10. Kinasewitz GT, Yan SB, Basson B, Comp P, Russell JA, Cariou A, Um SL, Utterback B, Laterre PF, Dhainaut JF: Universal changes in biomarkers of coagulation and inflammation occur in patients with severe sepsis, regardless of causative microorganism [ISRCTN74215569]. Crit Care 2004, 8:R82-R90.

11. Tamion F, Cam-Duchez V, Menard JF, Girault C, Coquerel A, Bonmarchand G: Erythropoietin and renin as biological markers in critically ill patients. Crit Care 2004, 8:R328-R335.

12. Charpentier J, Luyt CE, Fulla Y, Vinsonneau C, Cariou A, Grabar S, Dhainaut JF, Mira JP, Chiche JD: Brain natriuretic peptide: a marker of myocardial dysfunction and prognosis during severe sepsis. Crit Care Med 2004, 32:660-665.

13. Chua G, Kang-Hoe L: Marked elevations in $\mathrm{N}$-terminal brain natriuretic peptide levels in septic shock. Crit Care 2004, 8: R248-R250.

14. Spies CD, Nordmann A, Brummer G, Marks C, Conrad C, Berger G, Runkel N, Neumann T, Muller C, Rommelspacher $\mathrm{H}$, et al:: Intensive care unit stay is prolonged in chronic alcoholic men following tumor resection of the upper digestive tract. Acta Anaesthesiol Scand 1996, 40:649-656.

15. von Dossow, V, Schilling C, Beller S, Hein OV, von Heymann C, Kox WJ, Spies CD: Altered immune parameters in chronic alcoholic patients at the onset of infection and of septic shock. Crit Care 2004, 8:R312-R321.

16. Rusavy Z, Sramek V, Lacigova S, Novak I, Tesinsky P, Macdonald IA: Influence of insulin on glucose metabolism and energy expenditure in septic patients. Crit Care 2004, 8:R213-R220.

17. Vriesendorp TM, DeVries JH, Hulscher JB, Holleman F, van Lanschot JJ, Hoekstra JB: Early postoperative hyperglycaemia is not a risk factor for infectious complications and prolonged in-hospital stay in patients undergoing oesophagectomy: a retrospective analysis of a prospective trial. Crit Care 2004, 8: R437-R442.

18. Williams MD, Braun LA, Cooper LM, Johnston J, Weiss RV, Qualy RL, Linde-Zwirble W: Hospitalized cancer patients with severe sepsis: analysis of incidence, mortality, and associated costs of care. Crit Care 2004, 8:R291-R298.

19. Soares M, Fontes F, Dantas J, Gadelha D, Cariello P, Nardes F, Amorim C, Toscano L, Rocco JR: Performance of six severityof-illness scores in cancer patients requiring admission to the intensive care unit: a prospective observational study. Crit Care 2004, 8:R194-R203.

20. Lagoa CE, de Figueiredo LF, Cruz RJ, Jr, Silva E, Rocha e Silva: Effects of volume resuscitation on splanchnic perfusion in canine model of severe sepsis induced by live Escherichia coli infusion. Crit Care 2004, 8:R221-R228.

21. Goscinski G, Lipcsey M, Eriksson M, Larsson A, Tano E, Sjolin J: Endotoxin neutralization and anti-inflammatory effects of tobramycin and ceftazidime in porcine endotoxin shock. Crit Care 2004, 8:R35-R41.

22. Kirov MY, Kuzkov VV, Kuklin VN, Waerhaug K, Bjertnaes LJ: Extravascular lung water assessed by transpulmonary single thermodilution and postmortem gravimetry in sheep. Crit Care 2004, 8:R451-R458

23. Peristeris $P$, Clark BD, Gatti S, Faggioni R, Mantovani A, Mengozzi $M$, Orencole SF, Sironi M, Ghezzi P: N-acetylcysteine and glutathione as inhibitors of tumor necrosis factor production. Cell Immunol 1992, 140:390-399.

24. Hein OV, Ohring R, Schilling A, Oellerich M, Armstrong VW, Kox WJ, Spies C: N-acetylcysteine decreases lactate signal intensities in liver tissue and improves liver function in septic shock patients, as shown by magnetic resonance spectroscopy: extended case report. Crit Care 2004, 8:R66-R71.

25. Emet S, Memis D, Pamukcu Z: The influence of N-acetyl-Lcystein infusion on cytokine levels and gastric intramucosal pH during severe sepsis. Crit Care 2004, 8:R172-R179.

26. Bernard GR, Reines HD, Halushka PV, Higgins SB, Metz CA, Swindell BB, Wright PE, Watts FL, Vrbanac JJ: Prostacyclin and thromboxane A2 formation is increased in human sepsis syndrome. Effects of cyclooxygenase inhibition. Am Rev Respir Dis 1991, 144:1095-1101.

27. Memis D, Karamanlioglu B, Turan A, Koyuncu O, Pamukcu Z: Effects of lornoxicam on the physiology of severe sepsis. Crit Care 2004, 8:R474-R482.

28. Bruhn A, Hernandez G, Bugedo G, Castillo L: Effects of positive end-expiratory pressure on gastric mucosal perfusion in acute respiratory distress syndrome. Crit Care 2004, 8:R306R311.

29. Mier J, Leon EL, Castillo A, Robledo F, Blanco R: Early versus late necrosectomy in severe necrotizing pancreatitis. $\mathrm{Am}$ Surg 1997, 173:71-75

30. De Waele JJ, Hoste E, Blot SI, Hesse U, Pattyn P, de Hemptinne B, Decruyenaere J, Vogelaers D, Colardyn F: Perioperative factors determine outcome after surgery for severe acute pancreatitis. Crit Care 2004, 8:R504-R511.

31. Kerbaul F, Brousse M, Collart F, Pellissier JF, Planche D, Fernandez C, Gouin F, Guidon C: Combination of histopathological and electromyographic patterns can help to evaluate functional outcome of critical ill patients with neuromuscular weakness syndromes. Crit Care 2004, 8:R358-R366.

32. Granja C, Dias C, Costa-Pereira A, Sarmento A: Quality of life of survivors from severe sepsis and septic shock may be similar to that of others who survive critical illness. Crit Care 2004, 8 : R91-R98.

33. Poeze M, Ramsay G, Gerlach H, Rubulotta F, Levy M: An international sepsis survey: a study of doctors' knowledge and perception about sepsis. Crit Care 2004, 8:R409-R413. 